\title{
Estudio por impedancia y ruido electroquímico de la pasivación anódica del paladio en medio alcalino(*)
}

\author{
Esteban Miguel García-Ochoa* y Juan Genescá"
}

\begin{abstract}
Resumen Se estudia la formación de las especies oxidadas formadas en el paladio en medio alcalino durante el proceso de polarización anódica por medio de técnicas potenciostáticas, impedancia y ruido electroquímico. En este trabajo se hace especial énfasis en el análisis de las oscilaciones en corriente que se presentan durante la formación de los diferentes óxidos como una manifestación de su dinámica. Por medio del ánálisis del caos se pretende poder determinar los potenciales característicos de formación de cada uno de ellos.
\end{abstract}

Palabras clave Pasivación anódica. Paladio. Impedancia. Ruido electroquímico. Caos.

\section{EIS and electrochemical noise study of anodic passivation of palladium in alkaline medium}

\begin{abstract}
Anodic passivation of palladium in alkaline medium is studied by means of different electrochemical techniques like potentiodynamic polarization curves, electrochemical impedance spectroscopy and electrochemical noise. A 'chaotic analysis of electrochemical current noise signals (current oscillations) corresponding to different palladium oxides formed anodically seem to give information about the different potentials characteristics of palladium oxides formation.
\end{abstract}

Keywords Anodic passivation. Palladium. EIS. Electrochemical noise. Chaos.

\section{INTRODUCCIÓN}

La formación electroquímica de películas de óxidos en los metales nobles ha sido estudiada intensamente, principalmente por sus propiedades catalíticas, utilizando para ello una gran variedad de técnicas electroquímicas ${ }^{[1-3]}$, así como técnicas in situ como microbalanzas ${ }^{[4,5]}$ y elipsometría ${ }^{[6]}$ entre otras, y técnicas ex situ como lo es la difracción de rayos $\mathrm{X}^{[7]}$.

En el caso concreto del paladio se ha encontrado que los óxidos e hidróxidos de paladio(II) como el $\mathrm{PdO}, \mathrm{Pd}(\mathrm{OH})_{2}$ y el $\mathrm{PdO} \cdot \mathrm{H}_{2} \mathrm{O}$ son mucho más estables que los óxidos de paladio(IV), los cuales sólo pueden existir en condiciones muy oxidantes ${ }^{[8-12]}$. Generalmente, se ha determinado la existencia de los diferentes óxidos de paladio mediante pruebas de voltametría cíclica, identificando las diferentes especies formadas durante el proceso anódico por la reducción de las mismas durante el proceso catódico, dado que en el momento de su formación no se observan cambios significativos en la curva anódica al irse formando los diferentes óxidos. Esta situación se presenta más claramente en medios alcalinos como lo han demostrado, entre otros investigadores, Bolzán ${ }^{[13]}$ y Jeong et al. ${ }^{[14]}$.

Debido a la importancia que tiene poder detectar e identificar la formación de los diferentes óxidos del paladio durante la polarización anódica hasta valores del potencial correspondientes al desprendimiento de oxígeno, en este trabajo se estudiará el proceso de pasivación del paladio por medio de la obtención de las curvas potenciodinámicas de polarización anódica, la técnica de impedancia electroquímica y se hará énfasis principalmente en el análisis de las oscilaciones (fluctuaciones) en la corriente que se presentan durante la formación de los diferentes productos, como una manifestación de su dinámica.

Las oscilaciones en el potencial y/o la corriente que se pueden presentar durante una reacción electroquímica ya se habían observado en diferentes áreas, como en electrocatálisis ${ }^{[15,16]}$ y corrosión ${ }^{[17,18]}$ entre otras. Analizando estas señales se ha podido obtener información muy valiosa para el entendimiento del fenómeno electroquímico involucrado,

(•) Trabajo recibido el día 11 de enero de 1999 y aceptado en su forma final el 9 de diciembre de 1999.

(*) Instituto Mexicano del Petróleo. Coordinación de la Investigación y Planeación. Eje Central Lázaro Cárdenas - 152.07730 México D.F. (México).

(**) Dpto. Ingeniería Metalúrgica. Facultad Química. UNAM. Ciudad Universitaria. 04510 México D.F. (México). Actualmente de año sabático en la Facultat Ciències. Universitat Autònoma Barcelona. 08193 Bellaterra, Barcelona (España). 
como se aprecia, por ejemplo, en el trabajo de Li et $a l^{[19]}$ donde la amplitud de la señal en potencial tiene una relación directa con la formación de $\mathrm{FeCl}_{2}$ durante la disolución de hierro en un medio con cloruros, o en el trabajo de Dewald et al. ${ }^{[20]}$ que reconoce una frecuencia característica de $0,270 \mathrm{~Hz}$ durante la formación de acetato de cobre.

El estudio de estas señales en potencial y/o corriente puede hacerse desde el punto de vista de la teoría del caos, la cual permite realizar una estimación de la dinámica involucrada en el proceso $^{[21,22]}$. Bassett et al. ${ }^{[23-26]}$ aplicaron este criterio para el estudio de las reacciones electroquímicas durante la electrodisolución del cobre en medios ácidos, demostrando la naturaleza caótica de la señal electroquímica para así caracterizar la dinámica involucrada en el proceso.

La palabra caos da cuenta del aspecto imprevisible de un fenómeno, del cual se dice que es caótico. El comportamiento caótico de ciertos sistemas se explica como una consecuencia del valor que toman determinados parámetros de las ecuaciones no lineales que los describen. Una pequeña variación de estos valores da lugar a una alteración de la evolución que aumenta rápidamente con el tiempo. Así es difícil cualquier previsión y el comportamiento del sistema pasa a ser caótico.

En este trabajo se utiliza la información generada por la técnica de ruido electroquímico para, mediante el análisis del caos correspondiente, obtener información que ayude en la interpretación de la formación de los diferentes óxidos responsables de la pasivación del paladio.

\section{TÉCNICA EXPERIMENTAL}

Para poder realizar las pruebas electroquímicas se utilizó un portamuestras especial que permite exponer a la solución una superficie de $1 \mathrm{~cm}^{2}$ de una placa de paladio, contando con un contacto eléctrico aislado de la solución.

La muestra de paladio fue de alta pureza (Johnson-Matthey), la cual se pulió hasta alúmina 0,05 $\mu \mathrm{m}$, se desengrasó con acetona y se lavó con agua bidestilada.

El medio en el cual se llevaron a cabo las pruebas fue una solución de $\mathrm{NaOH} \mathrm{0,5} \mathrm{M} \mathrm{(pH} \mathrm{12,4)}$ a temperatura ambiente.

El equipo empleado fue una interfase electroquímica Solartron 1286. Este equipo controla el potencial del espécimen con respecto a un electrodo de referencia, que en este caso fue un electrodo de calomelanos saturado (ecs), utilizándose como contraelectrodo una barra de grafito. Dicha interfase electroquímica se comunicó a un ordenador personal por medio de un puerto serie (RS232). Con esta configuración se llevaron a cabo las pruebas potenciodinámicas y de registro de oscilaciones de corriente a diferentes potenciales con una velocidad de muestreo de 1,4 Hz. Para las pruebas de impedancia se acopló a esta configuración un analizador de respuesta en frecuencia Solartron 1253, con un barrido de frecuencias de $20.000 \mathrm{~Hz}$ a $0,05 \mathrm{~Hz}$ y una amplitud de $20 \mathrm{mV}$.

\section{RESULTADOS EXPERIMENTALES Y DISCU- SIÓN}

La figura 1 muestra las curvas de polarización anódica con barrido directo del potencial para el paladio en medio alcalino, $\mathrm{NaOH} 0,5 \mathrm{M}$, pudiéndose apreciar el efecto de la velocidad de barrido del potencial. En términos generales, al aumentar la velocidad de barrido se obtiene una mayor corriente, pero independientemente de este hecho se observan tres zonas, que especialmente en la curva obtenida a $8 \mathrm{mV} / \mathrm{s}$ toman la forma de inflexiones, una que correspondería al proceso de disolución del paladio y que tiene lugar aproximadamente entre los potenciales de -110 a $70 \mathrm{mV}$ (ecs); una segunda zona, entre 70 y $450 \mathrm{mV}$ (ecs), donde la densidad de corriente se mantiene sensiblemente constante y que corresponde al intervalo de potencial donde se esperaría la formación de los óxidos de paladio ${ }^{[27-29]} \mathrm{y}$, finalmente, en el intervalo de potencial entre 450 y $1.000 \mathrm{mV}$ (ecs), una tercera zona, en la que la densidad de corriente aumenta continuamente al ir incrementando el potencial

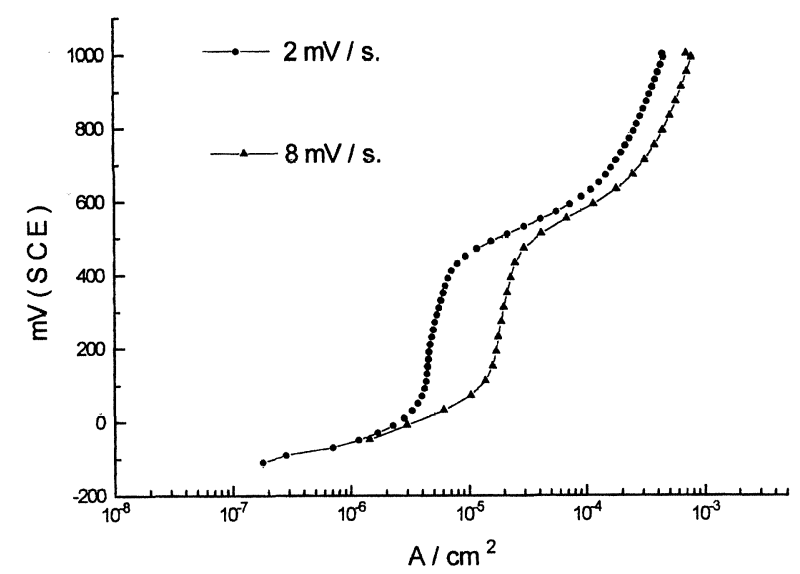

Figura 1. Curvas de polarización potenciodinámica anódica del paladio en $\mathrm{NaOH} 0,5 \mathrm{M}$.

Figure 1. Anodic polarization curve of paladium in $\mathrm{NaOH}$ $0.5 \mathrm{M}$. 
en la dirección anódica, la cual correspondería a la reacción de desprendimiento de oxígeno sobre la superficie oxidada del paladio.

En la figura 2 se presenta la curva de polarización del paladio en $\mathrm{NaOH} 0,5 \mathrm{M}$ a una velocidad de barrido más lenta de $2 \mathrm{mV} / \mathrm{s}$ con objeto de poder apreciar mejor las inflexiones que pudieran corresponder o ser causadas por la formación de los diferentes óxidos. Se puede apreciar, en el intervalo de potenciales comprendido entre 70 y $450 \mathrm{mV}$ (ecs), la presencia de tres cambios de pendiente. El primero, entre 100 y $190 \mathrm{mV}$ (ecs), podría corresponder a la formación de $\mathrm{PdO}$ que es el primer producto de oxidación, como fue señalado por Chausse et al. ${ }^{[27]}$. El segundo cambio de pendiente, entre 250 y $330 \mathrm{mV}$ (ecs), podría ser debido a la formación del $\mathrm{PdO}_{2}$ como fue reportado por Arvia et al. ${ }^{[30]}$. Finalmente, un tercer cambio de pendiente, entre 350 y $420 \mathrm{mV}$ (ecs), que podría atribuirse posiblemente a la formación de $\mathrm{PdO}_{3}$, especie propuesta por Burke et al. ${ }^{[31]}$. Estos cambios de pendiente podrían ser una indicación de la formación de diferentes especies químicas sobre el electrodo y aunque las pendientes son muy similares, con la

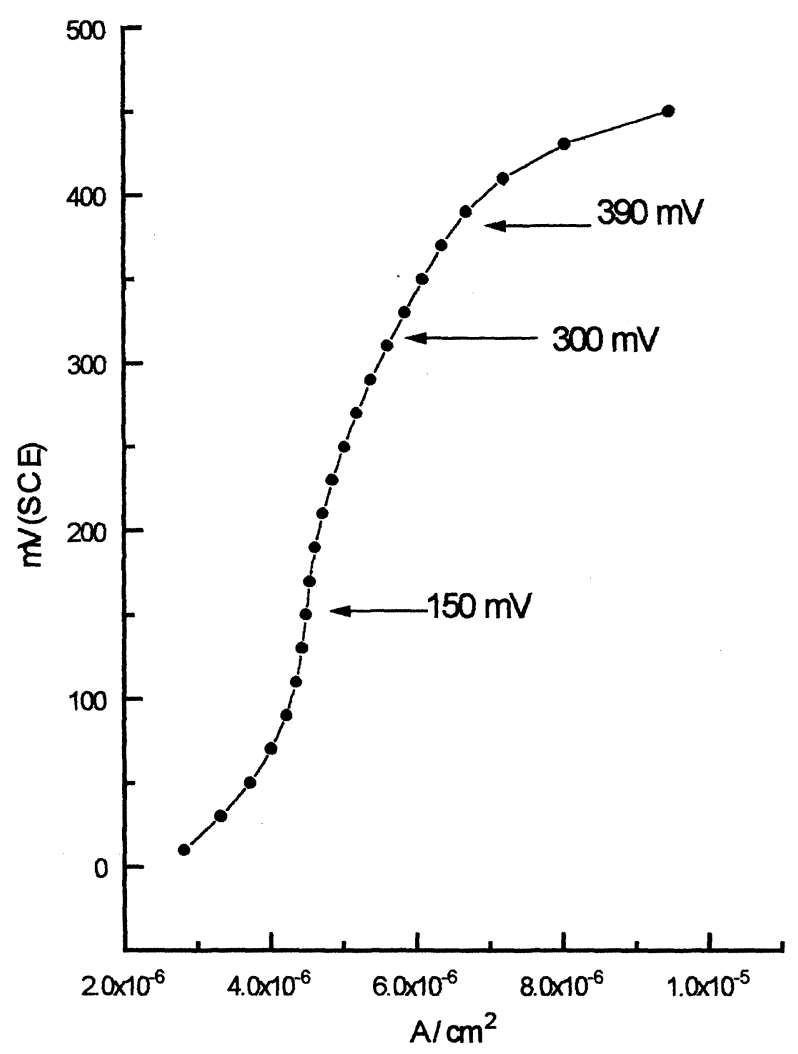

Figura 2. Curva de polarización potenciodinámica anódica del paladio en $\mathrm{NaOH}$ 0,5 M. Velocidad de barrido del potencial: $2 \mathrm{mV} / \mathrm{s}$.

Figure 2. Anodic polarization curve of paladium in $\mathrm{NaOH}$ $0.5 \mathrm{M}$. Potential sweep: $2 \mathrm{mV} / \mathrm{s}$. consiguiente dificultad de poderlas apreciar claramente, más adelante se presentarán evidencias experimentales más claras de estas especies por medio de otras técnicas electroquímicas y, principalmente, con el análisis de las oscilaciones de corriente.

La figura 3 corresponde a los diagramas de $\mathrm{Ny}$ quist del paladio a diferentes potenciales comprendidos dentro del intervalo de 0 a $150 \mathrm{mV}$ (ecs), obtenidos mediante la técnica de impedancia electroquímica. Se puede apreciar claramente la presencia de un solo semicírculo que incrementa su valor en la componente imaginaria al hacerse el potencial más positivo, pero manteniendo un mismo valor para la constante de tiempo de aproximadamente $1,87 \mathrm{~s}$.

En la figura 4 se presentan los diagramas de $\mathrm{Ny}$ quist de las pruebas de impedancia correspondientes

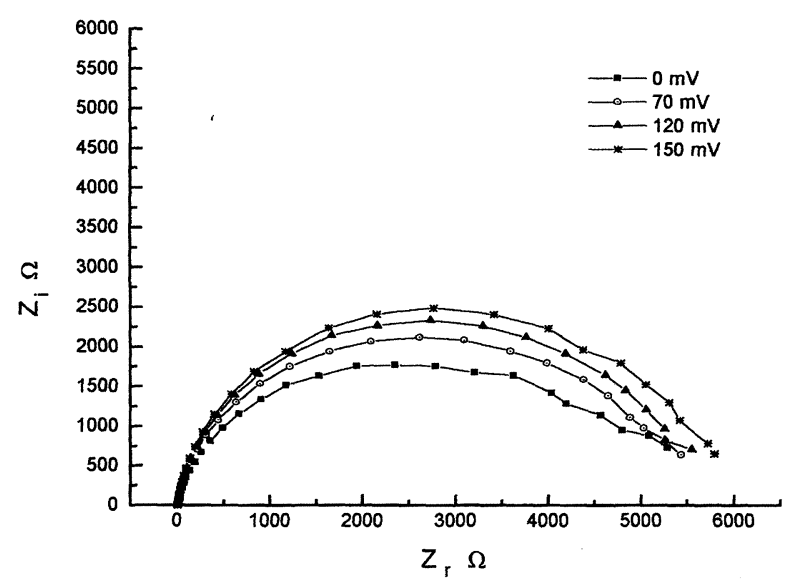

Figura 3. Diagramas de Nyquist del paladio en $\mathrm{NaOH} 0,5$ $\mathrm{M}$ correspondientes a diferentes potenciales de polarización.

Figure 3. Nyquist diagrams of paladium in $\mathrm{NaOH} 0.5 \mathrm{M}$ corresponding to different polarization potentials.

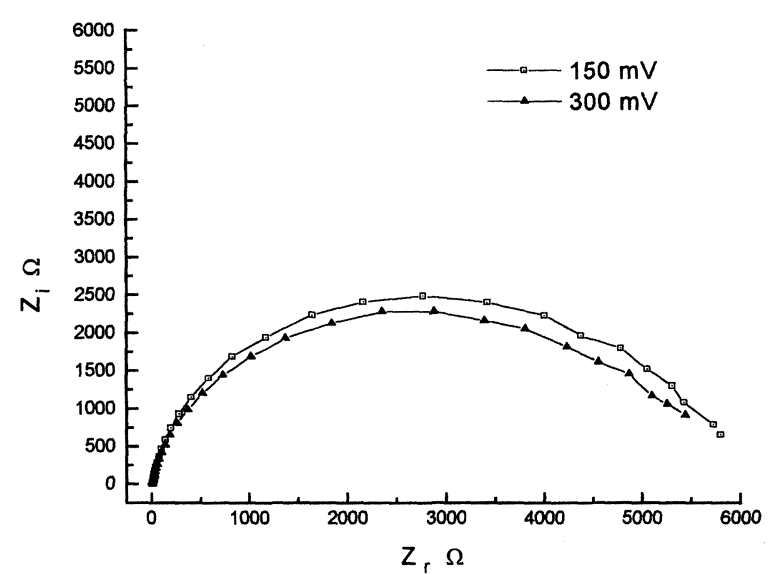

Figura 4. Diagramas de Nyquist del paladio en $\mathrm{NaOH} 0,5$ $\mathrm{M}$ correspondientes a 150 y $300 \mathrm{mV}$.

Figure 4. Nyquist diagrams of paladium in $\mathrm{NaOH} 0.5 \mathrm{M}$ corresponding to 150 and $300 \mathrm{mV}$ polarization potentials. 
a 150 y $300 \mathrm{mV}$ (ecs), pudiéndose observar la presencia de un solo semicírculo capacitivo, pero en esta ocasión disminuye el valor de la componente imaginaria al volverse el potencial más anódico, contrariamente a lo que sucedía en la figura 3, presentándose, además, dos valores de la constante de tiempo, $1,87 \mathrm{~s}$ para el caso de $150 \mathrm{mV}$ y $2,43 \mathrm{~s}$ para $300 \mathrm{mV}$. Como se puede apreciar, los dos valores son muy similares, lo que ocasiona que los diagramas sean muy parecidos.

La figura 5 corresponde a los resultados de impedancia obtenidos a 330 y $390 \mathrm{mV}$ (ecs). Los valores de impedancia, tanto en el eje real como imaginario, aumentan sustancialmente con respecto a los casos anteriores. En esta ocasión, los valores de las constantes de tiempo son mayores, 12,3 y $9,8 \mathrm{~s}$ para 330 y $390 \mathrm{mV}$, respectivamente.

Como en los casos anteriores, el diagrama de Nyquist presenta un solo semicírculo. Y si bien el corrrespondiente a $330 \mathrm{mV}$ parecería señalar la presencia de un segundo semicírculo, el diagrama de Bode pone de manifiesto que se trata de uno solo. Lo anterior podría deberse a un error experimental en el quinto punto, que induce a suponer, en la representación de Nyquist, un segundo proceso.

Finalmente, en la figura 6 se presentan los diagramas de impedancia a 600, 750 y $850 \mathrm{mV}$ (ecs), potenciales que corresponden a la zona de la curva de polarización donde se esperaría encontrar el proceso de desprendimiento de oxígeno. El diagrama de impedancia correspondiente a un potencial de $600 \mathrm{mV}$ exhibe un semicírculo capacitivo con una constante de tiempo de 0,033 s y un semicírculo inductivo con una constante de $0,340 \mathrm{~s}$, mientras que a los potenciales de 750 y $850 \mathrm{mV}$,

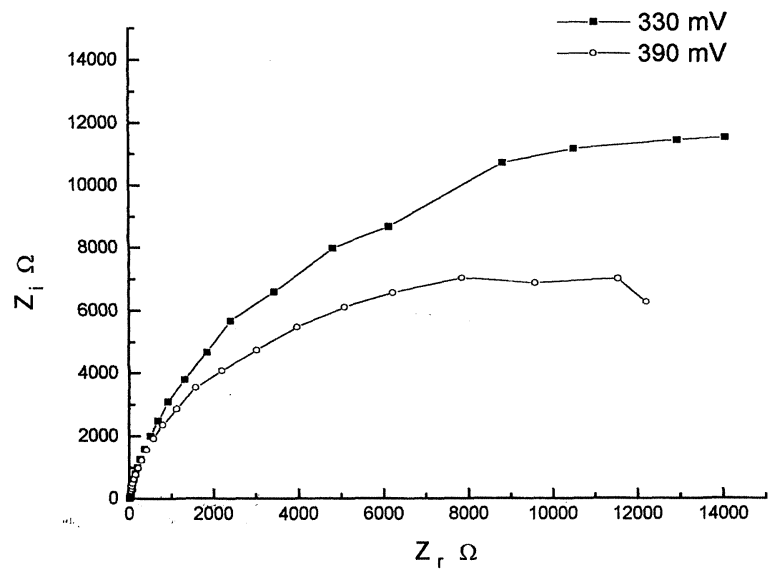

Figura 5. Diagramas de Nyquist del paladio en $\mathrm{NaOH} 0,5$ $M$ correspondientes a 330 y $390 \mathrm{mV}$.

Figure 5. Nyquist diagrams of paladium in $\mathrm{NaOH} \mathrm{0,5} \mathrm{M}$ corresponding to 330 and $390 \mathrm{mV}$ polarization potentials.

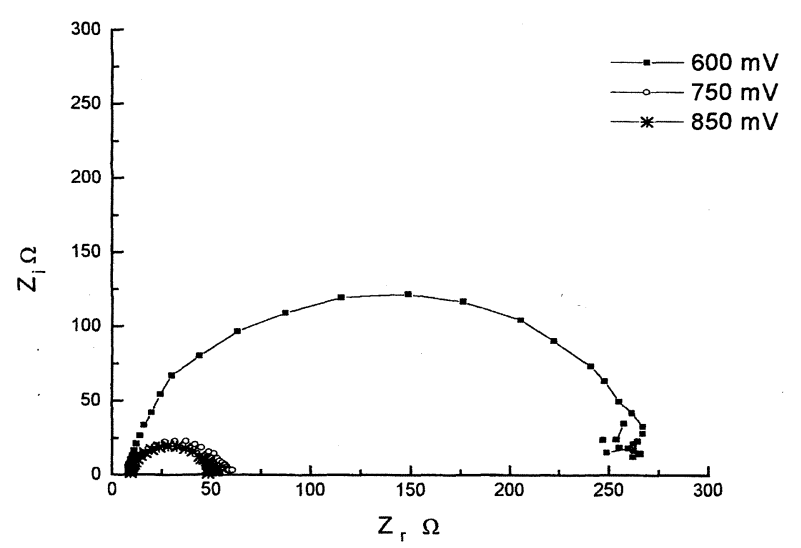

Figura 6. Diagramas de Nyquist del paladio en $\mathrm{NaOH} 0.5$ $M$ correspondientes a los potenciales 600,750 y $850 \mathrm{mV}$.

Figure 6. Nyquist diagrams of paladium in $\mathrm{NaOH} 0.5 \mathrm{M}$ corresponding to 600,750 and $900 \mathrm{mV}$ polarization potentials.

sólo se aprecia un semicírculo capacitivo donde las constantes de tiempo son de 0,0057 y 0,0043 s, respectivamente. Con relación a estos resultados se puede presumir que a los $600 \mathrm{mV}$ habría una adsorción de oxígeno que se reflejaría en el semicírculo inductivo y que el hecho de que los valores de impedancia, tanto en la parte real como imaginaria, desciendan significativamente, permite suponer a este potencial como un punto de transición entre la formación de los diferentes óxidos y el inicio de la reacción de formación de oxígeno sobre la superficie oxidada del paladio.

A potenciales superiores, 750 y $850 \mathrm{mV}$ (ecs), tomando como base los resultados experimentales de impedancia obtenidos, los cuales indican una reducción drástica de los valores de impedancia, tendría lugar, únicamente, la reacción de desprendimiento de oxígeno.

El análisis de los diagramas de impedancia se realizó buscando el circuito eléctrico equivalente que más se ajustara a los resultados experimentales obtenidos. Para ello, se utilizó el programa Zview de Scribner, pero siempre teniendo en cuenta que el circuito eléctrico propuesto tuviera un significado físico de acuerdo al sistema estudiado. Dado que se está estudiando el caso de un electrodo metálico, paladio, recubierto con un película de óxido y sumergido en un electrólito, se propone como modelo físico el compuesto por el sistema metal-óxido-electrólito, pudiendo esperarse, por tanto, las contribuciones en forma de capacitores de las interfases metal-óxido y óxido-electrólito, así como la propia de la película de óxido formado. Estas capacitancias estarían conectadas 
eléctricamente mediante resistencias, indicadoras éstas de la naturaleza defectuosa de las interfases y de la película de óxido. Naturalmente, debería considerarse la resistencia propia del electrólito. Con este planteamiento se obtuvieron los circuitos eléctricos equivalentes de cada uno de los diagramas obtenidos. Así, el correspondiente a la figura 3 es un circuito $\mathrm{RC}$ en paralelo y a su vez en serie con la resistencia del electrólito, Re, como sería de esperar para una interfase metal-electrólito. Este mismo circuito ajusta bien con los resultados experimentales para los diagramas de la figura 4 , mientras que en el caso de la figura 5, el mejor ajuste se obtiene con dos circuitos $\mathrm{RC}$ en paralelo y en serie entre sí y con la Re. Este segundo circuito RC podría corresponder conjuntamente a las interfases metal-óxido y óxido-electrólito. Por último, el circuito eléctrico equivalente a los diagramas de la figura 6, correspondiente a la zona de potenciales donde tiene lugar la formación de oxígeno, se vuelve muy complejo al necesitarse tres circuitos RC para lograr un buen ajuste.

El análisis por impedancia no parece aportar, pues, información relevante sobre el proceso de formación de los diferentes óxidos sobre el paladio, pareciendo que una película de óxido podría estar presente a partir de la aparición del segundo circuito $\mathrm{RC}$, lo cual tiene lugar a un potencial de 330 $\mathrm{mV}$ (ecs).

La presencia de oscilaciones en la corriente a diferentes potenciales son un reflejo de la dinámica de los procesos que se están llevando a cabo en la superficie del electrodo, ofreciendo la posibilidad de obtener información de los mismos. El tratamiento que se les aplicó a las series de tiempo en corriente a diferentes potenciales consistió en remover la tendencia ${ }^{[32]}$ que presentaban y obtener las fluctuaciones medias para no tener una contribución exagerada de las componentes en baja frecuencia que pudieran ocultar valores de otras. A las mismas series se les aplicó un filtro de paso de bajas de Butterworth ${ }^{[33]}$ de orden de $9^{\circ}$, para contar solamente con las frecuencias en el intervalo de 0 a $100 \mathrm{mHz}$, dado que originalmente se contaba con un intervalo de frecuencias de 0 a $700 \mathrm{mHz}$ debido a la velocidad de muestreo. El objeto de la aplicación de este filtro fue en primer lugar estar en un intervalo de frecuencias típico en el que se pudiera apreciar la formación de productos debido a las reacciones electroquímicas ${ }^{[34]}$, así como minimizar el ruido presente en las mismas para una mejor aplicación del análisis de caos, como fue recomendado por Wolf et al. ${ }^{[35]}$.
En la figura 7 se muestran las series de tiempo a diferentes potenciales correspondientes a la zona de disolución, pasivación y desprendimiento de oxígeno. Se aprecia claramente que en las series de tiempo, tanto en la zona de disolución como de pasivación, las señales son muy semejantes, tanto en forma como en magnitud, y sólo la que corresponde al desprendimiento de oxígeno tiene una apariencia diferente. Esto se ve confirmado en la figura 8 en la que se presentan los análisis espectrales de las series de tiempo anteriores. Como puede comprobarse, en todo el espectro de frecuencias no existe una sola que sobresalga de las demás en forma importante y sólo en el intervalo que corresponde al desprendimiento de oxígeno se aprecia claramente una contribución muy importante de las frecuencias comprendidas entre 1 y 6 $\mathrm{mHz}$.

Como resultado de este análisis espectral queda de manifiesto que no se observan diferencias significativas en la formación de los diferentes óxidos de paladio, lo que hace necesario recurrir a otro tipo de análisis, como el del caos, para las diferentes series de tiempo. Primeramente, se debe determinar si las señales que se están analizando son de naturaleza caótica. Para ello es necesario determinar el valor de la entropía de Kolmogorov $(K)$ que no es otra cosa que la tasa media de ganancia de la información ${ }^{[36]}$. Un valor de $K=0$ corresponde a un sistema determinístico, cuando $K$ es infinito se tiene un proceso aleatorio $y$, finalmente, cuando $K$ es una constante positiva y diferente de cero corresponde a un proceso caótico ${ }^{[37]}$. La obtención de este valor de entropía $(K)$ es relativamente sencillo cuando se trabaja sobre modelos analíticos definidos, pero no es así para cuando se quiere estimarla en una serie de tiempo. Grassberger et al. ${ }^{[37]}$ propusieron un método para superar esta limitación el cual se empleó en este trabajo para estimar el valor de $K$ en las series de tiempo en corriente a diferentes potenciales.

La figura 9 muestra el valor estimado de $K$ para cada una de las series de tiempo, pudiéndose apreciar que para todos los potenciales, el valor de $K$ es una constante positiva, lo cual indica que el fenómeno en cada potencial es de naturaleza caótica. Algo muy significativo que se aprecia en esta figura es la presencia de dos zonas, la primera comprendida desde -130 a $550 \mathrm{mV}$ (ecs), que corresponde al intervalo de potenciales donde se forman los diferentes óxidos de paladio, en la que $K$ tiene un valor promedio de 0,42 , y la segunda, desde 600 a $1.000 \mathrm{mV}$ (ecs), que corresponde al intervalo de 

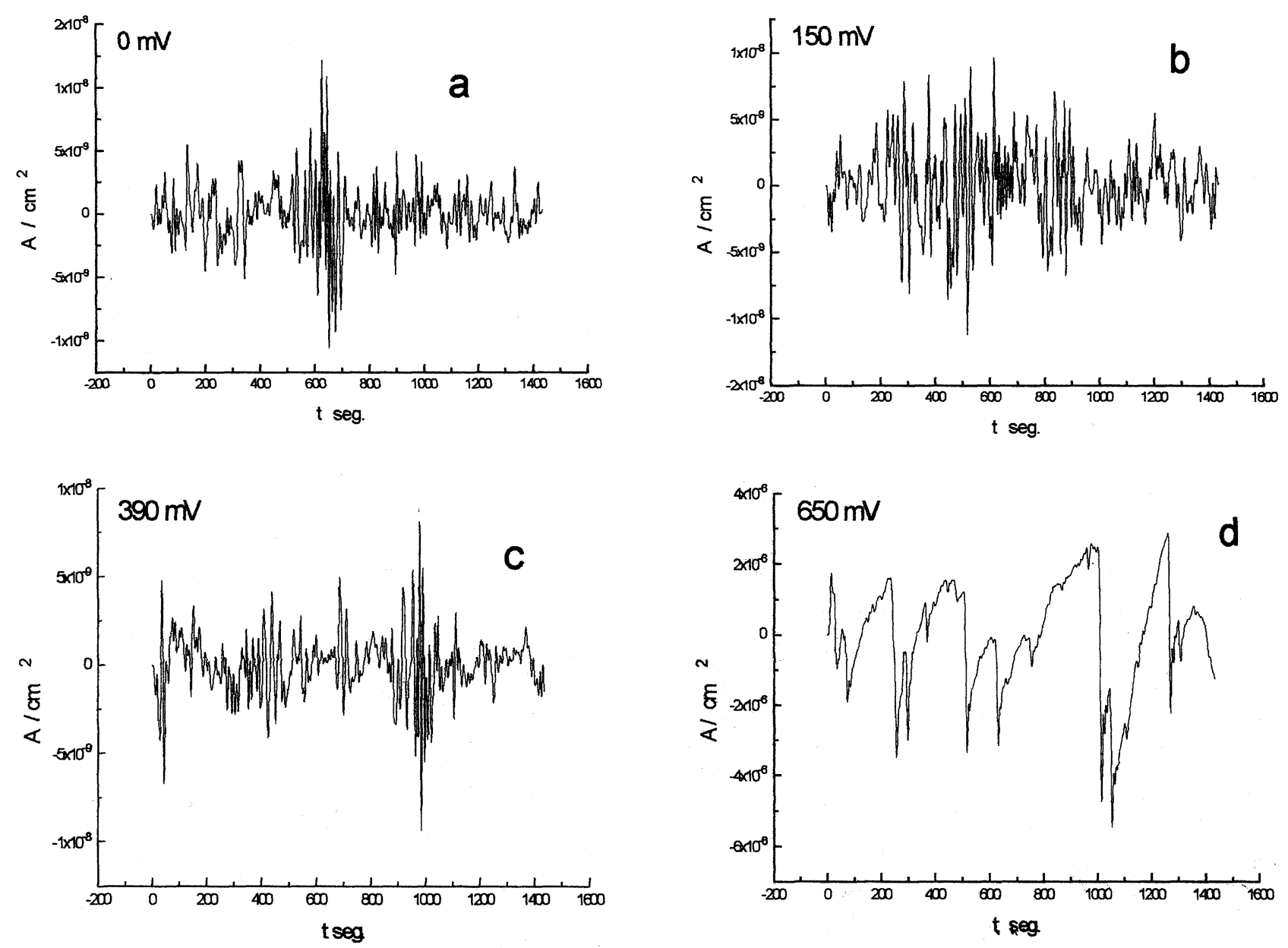

Figura 7. Series de tiempo del ruido electroquímico correspondientes al paladio en $\mathrm{NaOH} 0,5 \mathrm{M}$ para diferentes potenciales de polarización.

Figure 7. Time series of current noise of paladium in $\mathrm{NaOH} 0.5 \mathrm{M}$ at different polarization potentials.

potenciales donde tiene lugar el desprendimiento de oxígeno, con un valor promedio de $K$ de 0,23 . Esto parece sugerir que la formación de los óxidos de paladio es de naturaleza más caótica que el proceso de desprendimiento de oxígeno.

De la observación de la figura 9 puede desprenderse que los valores calculados de la entropía de Kolmogorov presentan una clara dispersión, mostrando sólo, en forma cualitativa, dos niveles de caos, uno para la formación de los óxidos del paladio y otro para la reacción de desprendimiento de oxígeno. Sólo se puede observar un ordenamiento cuando se determina el coeficiente de Lyapunov más positivo (Fig. 10), que es en sí la parte más significativa de la divergencia o de la entropía de Kolmogorov, mostrando claramente una serie de mínimos que están en función de las especies de paladio reportadas.

La presencia de mínimos en el coeficiente de Lyapunov en un sistema dinámico no es extraño, pudiéndose apreciar esto en la siguiente ecuación dinámica que es extremadamente sencilla:

$$
X_{n+1}=A X_{n}\left(1-X_{n}\right)
$$

donde el parámetro A determina el nivel de caos, el cual puede asociarse al potencial. Este potencial es característico de la formación de alguna especie química en particular y cuando existe la posibilidad de formación de una nueva especie y, por tanto, se puede establecer una competencia, aumentando la complejidad del sistema, el nivel de caos debe aumentar. De aquí la importancia de proponer un análisis no lineal.

Otro parámetro muy importante, pues, en la teoría de caos es el denominado exponente o coeficiente de Lyapunov, que es la rapidez en que dos trayectorias cercanas en el espacio de fase divergen o más simplemente, el promedio de la pérdida de la información entre puntos vecinos. En un sistema dinámico en un espacio de fase $n$-dimensional existirán tantos coeficientes de Lyapunov 

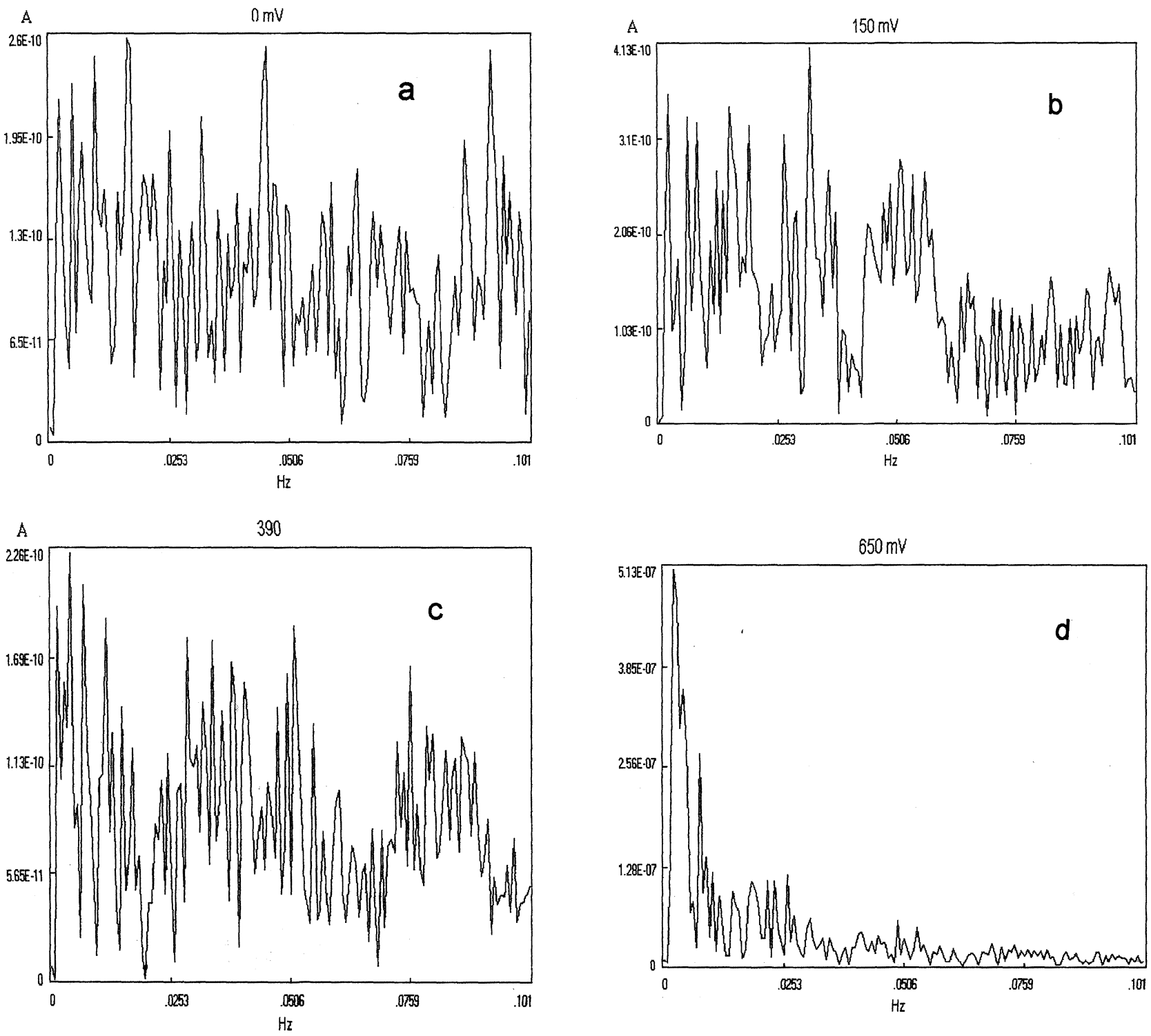

Figura 8. Análisis espectral correspondiente a las series de tiempo de la figura 7.

Figure 8. Spectral analysis of time series showed at figure 7. como dimensiones en las cuales esté contenido el atractor. Esto puede expresarse matemáticamente de la siguiente forma:

$$
\lambda_{1}=\lim _{t \rightarrow \infty} \frac{1}{t} \ln \frac{p_{\mathrm{i}}(t)}{p_{\mathrm{i}}(0)}
$$

donde $p_{\mathrm{i}}(t)$ es el eje principal de una elipsoide ${ }^{[35]}$.

Dado que un exponente de Lyapunov positivo corresponde a la sensibilidad que tiene un sistema a las condiciones iniciales o a la velocidad de generación de la información, se puede llegar a establecer que la suma de los exponentes positivos de Lyapunov corresponde al valor de la entropia de Kolmogorov $^{[36]}$, lo cual, matemáticamente, se expresaría como,

$$
K=\sum_{\lambda_{i} \geq 0} \lambda_{i}
$$

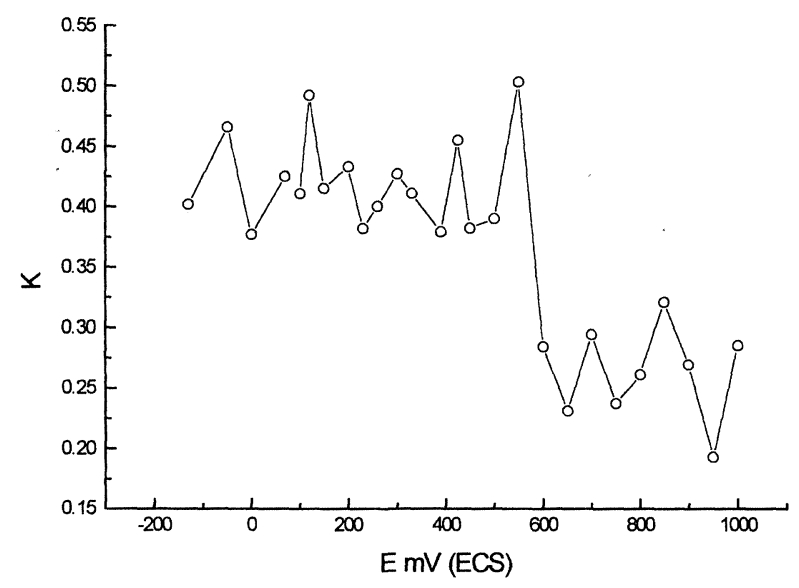

Figura 9. Valores de la entropia de Kolmogorov para paladio en $\mathrm{NaOH} 0,5 \mathrm{M}$ a diferentes potenciales de polarización.

Figure 9. Kolmogorov entropy values for paladium in $\mathrm{NaOH} 0.5 \mathrm{M}$ at different polarization potentials. 


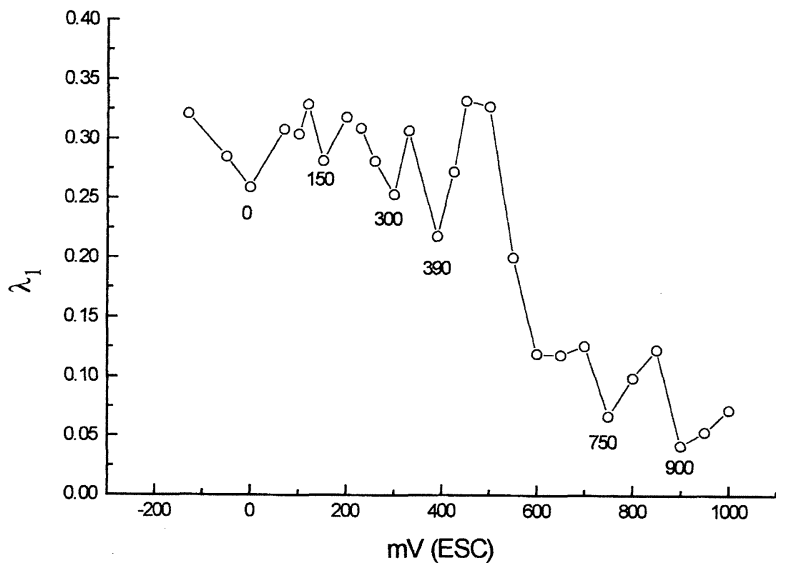

Figura 10. Valores de los exponentes más positivos de Lyapunov para diferentes potenciales de polarización. Paladio en $\mathrm{NaOH} 0,5 \mathrm{M}$.

Figure 10. Maximum (Largest) Lyapunov exponents at different polarization potentials. Paladium in $\mathrm{NaOH} 0.5 \mathrm{M}$.

Esto supondría que sólo sería necesario un exponente de Lyapunov positivo para que el sistema fuera considerado como caótico.

El exponente de Lyapunov más significativo para establecer lo caótico de un sistema es precisamente el más positivo $\left(\lambda_{1}\right)$, el cual se puede expre-

$$
\lambda_{1}=\lim _{\mathrm{N} \rightarrow \infty} \frac{1}{N t_{\mathrm{s}}} \sum_{\mathrm{i}=1}^{\mathrm{N}} \ln \left[\frac{L\left(t_{\mathrm{i}}\right)}{L\left(t_{\mathrm{i}-1}\right)}\right]
$$

sar de la siguiente forma para una serie de tiempo: donde $L\left(t_{\mathrm{i}}\right)$ es la distancia entre dos trayectorias en un tiempo determinado, después de una pequeña evolución en el tiempo, $t_{\mathrm{s}}$. Esto se repite $t_{\mathrm{i}}=i t_{\mathrm{s}}(i$ $=1,3 \ldots \mathrm{N})$, donde una trayectoria es reemplazada por la calculada. El coeficiente de Lyapunov más positivo $\left(\lambda_{1}\right)$ se obtiene por el promedio sobre el mayor número de tales reemplazos ${ }^{[35-38]}$.

Rosenstein et al. ${ }^{[39]}$ propusieron una metodología para la estimación del exponente más positivo de Lyapunov $\left(\lambda_{1}\right)$ a través de un algoritmo rápido, fácil de implementar y robusto en el cambio de las siguientes propiedades: dimensión embebida, tamaño de la serie de tiempo y el nivel de ruido. Este algoritmo se empleó en este trabajo para la evaluación de $\lambda_{1}$ en las series de tiempo experimentales.

La figura 10 muestra los valores de $\lambda_{1}$ para las series de tiempo en corriente a diferentes potenciales, apreciándose nuevamente que existen dos secciones en la gráfica, una que corresponde a la formación de los diferentes óxidos de paladio y otra a la formación de oxígeno, como en el caso de la figura 9, donde se expresan los valores de $\mathrm{K}$. Pero en esta ocasión quedan muy bien definidos los valores mínimos a 0, 150, 300 y $390 \mathrm{mV}$ (ecs) en la sección que corresponde al intervalo de potencial de -130 a $500 \mathrm{mV}$ (ecs), y para el intervalo de potencial de 600 a $1.000 \mathrm{mV}$ (ecs) existen dos mínimos, a 750 y a $900 \mathrm{mV}$ (ecs).

Tomando como base los valores obtenidos de $\lambda_{1}$ en el intervalo de potencial de -130 a $500 \mathrm{mV}$ se puede proponer que los valores mínimos obtenidos podrían tener relación con la formación de las diferentes especies químicas, estando esto apoyado por el hecho de que cuando dos especies compiten por su formación, el nivel de caos se incrementa y, obviamente, cuando esta competencia no existe, el nivel de caos disminuye, con excepción del valor de $0 \mathrm{mV}$ que pudiera estar relacionado con la formación de $\mathrm{PdOH}$ que es un precursor de la formación del $\mathrm{PdO}$ como fue establecido por Bolzán ${ }^{[13]}$ de acuerdo al siguiente mecanismo:

$$
\mathrm{PdOH} \leftrightarrow \mathrm{PdO}^{*} \rightarrow \mathrm{PdO}
$$

El valor de $150 \mathrm{mV}$ pudiera esta relacionado con la formación de $\mathrm{PdO}$, el de $300 \mathrm{mV}$ con la de $\mathrm{PdO}_{2}$ y, finalmente, el de $390 \mathrm{mV}$ con la formación de $\mathrm{PdO}_{3}$.

Los valores mínimos de 750 y $900 \mathrm{mV}$, en el intervalo de potencial de 600 a $1.000 \mathrm{mV}$, que corresponde al desprendimiento de oxígeno, pudieran estar asociados a dos mecanismos diferentes para esta reacción, que entrarían en competencia, pero esto sería tema de otro trabajo de investigación.

Finalmente, la figura 11 es una representación en dos dimensiones del atractor obtenido en cada serie de tiempo, donde se representó gráficamente $X$ que es el valor en cada instante de la variable que se está midiendo, es decir la corriente, contra su derivada $\left(X^{\prime}\right)^{[22]}$, pudiéndose interpretar como una "imagen" de la dinámica del sistema para cada uno de los potenciales estudiados, ya que permite seguir los cambios en el valor de la derivada de la corriente con respecto al tiempo, como forma de expresión de la dinámica de la misma. La obtención del atractor consiste en representar gráficamente la pendiente $X^{\prime}$ contra cada punto $X$ de la serie de tiempo. $\mathrm{Pa}$ ra obtener la pendiente que le corresponde a cada punto de la serie se asume que la mitad de la diferencia entre dos puntos adyacentes corresponde a la derivada para cada punto. Se puede apreciar que se trata de un mismo atractor, pero con ciertas modificaciones, propias de los cambios debidos al potencial para los valores de 0,150 y $390 \mathrm{mV}$ y sólo el que 

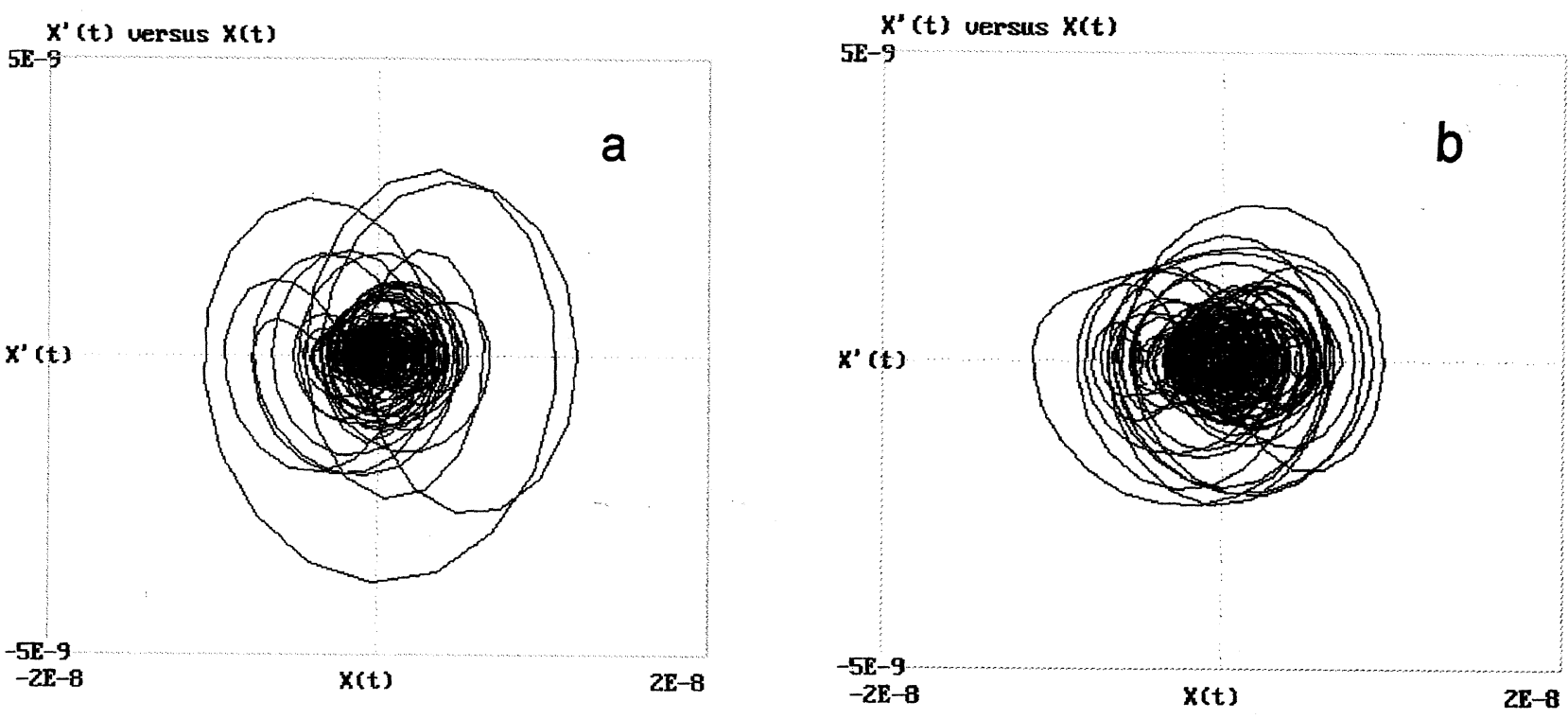

$x^{\prime}(t)$ versus $x(t)$
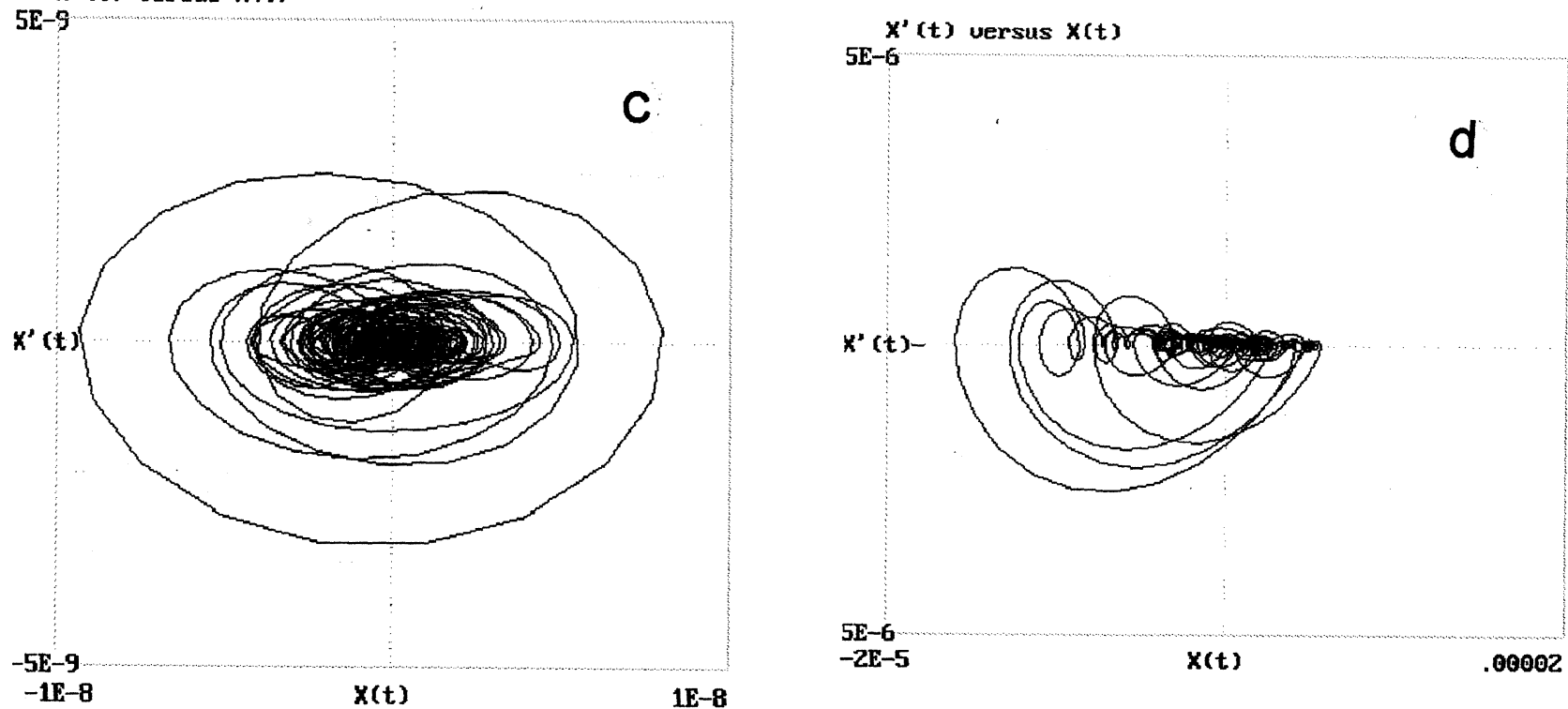

Figura 11. Representación gráfica del atractor obtenido para cada serie de tiempo de la figura 7.

Figure 11. Two dimensional projections of electrochemical noise attractor generated at the different polarization potentials showed in figure 7.

corresponde a $650 \mathrm{mV}$ es un atractor diferente, que es el que representa la dinámica del proceso de evolución de oxígeno.

\section{CONCLUSIONES}

Se pudo determinar que la formación de los diferentes óxidos de paladio como resultado de la polarización anódica en un medio alcalino presenta una dinámica muy similar, pudiendo esto explicar la poca efectividad de las técnicas potenciostática y de impedancia para determinar y diferenciar los óxidos formados durante la pasivación anódica.
El análisis mediante la aplicación de la teoría del caos a las señales de ruido electroquímico en corriente fue en este caso determinante, porque mostró claramente que las mismas eran de naturaleza caótica y no aleatoria, poniendo de manifiesto que la dinámica de formación de los diferentes óxidos es multiperiódica. La determinación del exponente de Lyapunov más positivo, $\lambda_{1}$, mostró valores mínimos en el intervalo de formación de los diferentes óxidos de paladio, haciendo presumir que a esos valores de potencial la especie formada es un óxido en especial, circunstancia que no pudo ser determinada ni por la técnica potenciostática ni por la de impedancia. 


\section{REFERENCIAS}

[1] A.E. Bolzan y A.J. ArviA, J. Electroanal. Chem. 354 (1993) 243-253.

[2] L.D. BuRKE y J.K. CASEY, J. Appl. Electrochem. 23 (1993) 573-582.

[3] L.D. BURKE Y J.K. CASEY, J. Electrochem. Soc. 140 (1993) 1284-1298.

[4] S. Bourkane, C. Gabriell y M. Keddam, Electrochim. Acta 38 (1993) 1827-1835.

[5] V.I. BRISS, H. Elzanowska y S. Gottesfeld, J. Electroanal. Chem. 318 (1991) 327-333.

[6] J.T. Remillard, W.H. Weber, J.R. McBride y R.E. Soltis, Appl. Phys. 71 (1992) 4515-4526.

[7] M. Fleischman y M.W. MaO, J. Electroanal. Chem. 229 (1987) 125-139.

[8] V.S. Bagotzky y M.R. TaRaseVICH, J. Electroanal. Chem. 101 (1979) 1-9.

[9] K. Gossner y E. Mizera, J. Electroanal. Chem. 125 (1991) 347-352.

[10] T. ChierChie, C. MAYer y W.J. LoRenz, J. Electroanal. Chem. 135 (1982) 211-220.

[11] A.E. BolzAN, M.E. Martins y A.J. ARVIA, J. Electroanal. Chem. 172 (1984) 221-233.

[12] A.E. BolzAN, M.E. MARTINS y A.J. ARVIA, J. Electroanal. Chem. 157 (1983) 339-358.

[13] A.E. BolzAN, J. Electroanal. Chem. 380 (1995) 127-138.

[14] M.C.Jeong, C.H. PYun e I.H. YeO, J. Electrochem. Soc. 140 (1993) 1986-1989.

[15] J. Wojtowicz, N. Marincic y B.E. Conway, J. Chem. Phys. 48 (1968) 4333-4341.

[16] H. TriButSCH, Phys. Chem. 79 (1975) 570-581.

[17] J. Uruchurtu y J.L. DaWSON, Corrosion 43 (1987) 19-25.

[18] J.J. PODESTA, R. PIATI y A.J. ARVIA, Electrochim. Acta 24 (1979) 633-637.

[19] W. LI, X. WANG y K. NoBE, J. Electrochem. Soc. 137 (1990) 1184-1188.

[20] H.D. Dewald, P. Parmananda y R.W. Rollins, J. Electrochem. Soc. 140 (1993) 1969-1973.
[21] J.T. SANDEFUl, Discrete Dynamical System, Clarendon Press, Oxford (Reino Unido) 1990.

[22] D. Gullck, Encounters With Chaos, McGraw-Hill, New York (EE.UU.), 1992.

[23] M.R. Basset y J.L. Hudson, J. Phys. Chem. 93 (1989) 2731-2737.

[24] M.R. BAsSett y J.L. Hudson, J. Electrochem. Soc. 137 (1990) 1815-1826.

[25] M.R. BASSET y J.L. HudSON, J. Electrochem Soc. 137 (1990) 922-932.

[26] M.R. Basset y J.L. Hudson, J. Phys. Chem. 92 (1988) 6963-6966.

[27] V. Chausse, P. Regull y L. Victori, J. Electroanal. Chem. 238 (1987) 115-128.

[28] J. Pares, P. Regull y L. Victorl, Afinidad 45 (1988) 105.

[29] J. Genesca y L. Victori, Rev. Coat. Corros. 4 (1981) 325-348.

[30] A.E. Bolzman, M.E. Martins y A.J. Arvia, J. Electroanal. Chem. 157 (1983) 339-358.

[31] L.D. BURKE y M.B.C. ROCHE, J. Electroanal. Chem. 186 (1985) 139-154.

[32] J. URUCHUTU, Tesis Doctoral, Universidad de Manchester, Reino Unido, 1981.

[33] R. KUC, Introduction to Digital Signal Processing, McGraw-Hill, New York, EE.UU., 1982.

[34] E. García, Tesis Doctoral, Universidad Nacional Autónoma de México, México D.F., 1994.

[35] A. Wolf, J.B. SWIFt, H. SWInNeY y J.A. VAStano, Physica D 16 (1985) 285-317.

[36] J.P. Eckman y D. Ruelle, Rev. Mod. Phys. 57 (1985) Part I. 617-656.

[37] P. Grassberger e I. Procaccia, Phys. Rev. A 28 (1983) 2591-2593.

[38] A. Legat y V. Dolecek, J. Electrochem. Soc. 142 (1995) 1851-1858.

[39] M.Y. Rosenstein, J.J. Coluns y C.J. De LuCA, Physica D 65 (1993) 117-134. 